\title{
The first large-scale genetic analysis of the vulnerable noble crayfish Astacus astacus reveals low haplotype diversity in central European populations
}

\author{
A. Schrimp $f^{(1)}$, H.K. Schulz ${ }^{(1)}, K$. Theissinger ${ }^{(1)}$, L. Pârvulescu(2), R. Schulz ${ }^{(1)}$ \\ Received January 14, 2011 \\ Revised June 6, 2011 \\ Accepted July 11, 2011
}

\section{ABSTRACT}

Key-words:

mtCOI

sequences,

haplotype

diversity,

human

translocation,

conservation

management,

evolutionary

significant units

(ESU)

\begin{abstract}
Major global changes (e.g., human impact or climatic cycles) have a severe impact on the distribution and diversity of species such as the vulnerable European noble crayfish Astacus astacus. This is the first large-scale study regarding haplotype diversity of $A$. astacus in central and southeastern Europe. We analyzed a partial sequence of the mitochondrial gene cytochrome oxidase subunit I from 416 specimens of 92 crayfish stocks of three European river basins (Black Sea, North Sea and Baltic Sea). Twentytwo haplotypes were identified, and one common haplotype was found across the whole study area. We detected differences in the genetic diversity between major river catchments ( $\Phi_{\mathrm{ST}}: 0.03481$ to 0.20387$)$. The high haplotype diversity $\left(H_{\mathrm{D}}=0.794 \pm 0.024\right)$ and high number of private haplotypes suggests a glacial refuge in the Balkan area. The very low haplotype diversity in central Europe $\left(H_{D}=0.299 \pm 0.038\right.$ and $\left.H_{D}=0.163 \pm 0.058\right)$ could be a result of human translocation and/or founder effects due to postglacial re-colonization. Nevertheless, the high frequency of private haplotypes in all major catchment areas indicates a differentiation of noble crayfish populations throughout Europe despite the extensive human translocation of crayfish. The results of this study support the establishment of conservation management plans for this vulnerable species.
\end{abstract}

\section{RÉSUMÉ}

Une première analyse génétique à grande échelle des populations vulnérables de l'écrevisse Astacus astacus révèle une faible diversité des haplotypes dans les populations du centre de l'Europe

Mots-clés :
séquences
mtCOI,
diversité des
haplotypes,
translocation,
gestion de la
conservation,
evolutionary
significant units
(ESU)

D'importants changements globaux (par exemple, l'impact humain ou les cycles climatiques) ont un grave impact sur la distribution et la diversité des espèces telle que l'écrevisse à pattes rouges européenne Astacus astacus. C'est la première étude à grande échelle en matière de diversité des haplotypes d'A. astacus en Europe centrale et du sud-est. Nous avons analysé une séquence partielle du gène mitochondrial de la cytochrome oxydase, sous-unité I de 416 spécimens de 92 stocks d'écrevisses de trois bassins hydrographiques européens (Mer Noire, Mer du Nord et Mer Baltique). Vingt-deux haplotypes ont été identifiés, et un haplotype commun a été trouvé dans toute la zone d'étude. Nous avons détecté des différences dans la diversité génétique entre les principaux bassins versants $\left(\Phi_{\mathrm{ST}}: 0,03481\right.$ à 0,20387$)$. La forte diversité des haplotypes $\left(H_{\mathrm{D}}=0,794 \pm\right.$ $0,024)$ et le nombre élevé d'haplotypes suggèrent un refuge glaciaire dans la région

(1) Institute for Environmental Sciences, University Koblenz-Landau, Fortstrasse 7, 76829 Landau, Germany, schrimpf@uni-landau.de

(2) Faculty of Chemistry, Biology and Geography, University of Timisoara, Timisoara, Romania 
des Balkans. La très faible diversité des haplotypes en Europe centrale $(0,299 \pm$ $H_{\mathrm{D}}=0,038$ et $\left.0,163 \pm H_{\mathrm{D}}=0,058\right)$ pourrait être une conséquence de la translocation de l'homme et / ou des effets fondateurs en raison de la re-colonisation postglaciaire. Néanmoins, la fréquence élevée des haplotypes particuliers dans tous les grands bassins versants indique une différenciation des populations d'écrevisses à pattes rouges à travers l'Europe, malgré les translocations importantes de l'écrevisse. Les résultats de cette étude viennent appuyer la mise en place de plans de conservation pour cette espèce vulnérable.

\section{INTRODUCTION}

Genetic diversity among and within species is the basis of evolution and is important for the resilience of native stocks to future changes in the environment (Östergren, 2006). The convention on biological diversity (CBD) highlighted the importance of genetic diversity within species as one of the three levels of biological diversity (CBD, adopted 1992). Therefore, the identification of genetically differentiated populations is crucial for the conservation and management of a species or the regional strains within a species. The guidelines for the protection of threatened species recommend the identification of evolutionary significant units (ESU, Ryder, 1986). An ESU may be defined as a population or group of populations that is sufficiently differentiated and requires a separate management or conservation strategy (Moritz, 1994). Defining management units can aid in selecting a management program among the various populations so that the greatest overall diversity is sustained by the conservation plan (Crozier, 1997). Nevertheless, conservation managers typically do not use genetic evidence (i.e., the identification of ESUs) to support their adopted measures (Pullin et al., 2004).

The IUCN red list of threatened species cites Astacus astacus as vulnerable with a decreasing population status (Edsman et al., 2010). A. astacus is widely distributed across Europe. Its range extends from Russia and the Ukraine in the east, Scandinavia in the north, Greece in the south, and the United Kingdom and France in the west (Edsman et al., 2010). Based on morphological criteria, different and partly contradictory classifications of the noble crayfish subspecies can be found in the literature (see Smietana et al., 2006 for a review). Here, we focus on the taxonomic studies of Karaman (1962) and Albrecht (1983). They described three subspecies with distinct geographical distributions: (1) $A$. a. colchicus is endemic to an isolated area in the Ponto-Caspian region of the upper Rion (Caucasus). (2) A. a. balcanicus inhabits the western Balkans in the drainage system of the Vardar river in Macedonia and Greece, as well as Lake Ohrid. (3) A. a. astacus inhabits the Danube river and its tributaries, as well as the rivers that open out into the North and Baltic Seas.

The fragmented nature of $A$. astacus' typical freshwater habitats (streams, rivers and lakes with sufficient water quality and an excess of shelter) coupled with a low migratory potential reduces the possibility of reestablishing local populations that have gone extinct (Abrahamsson, 1971; Strayer and Dudgeon, 2010). In addition to environmental contributions to the decreasing number of stocks (i.e., water pollution or channelization of streams), the oomycete Aphanomyces astaci is decreasing A. astacus' numbers (Schikora, 1906). This invasive pathogen was brought to Europe via American crayfish species (e.g., Orconectes limosus) in the mid-nineteenth century (Alderman et al., 1990) and caused massive die-offs in native crayfish stocks (Alderman, 1996).

During the Middle Ages, the noble crayfish became a commercial object throughout Europe, and it has been sold as a delicacy ever since (Schulz et al., 2004). The translocation of individuals and whole stocks (Skurdal and Taugbøl, 2002), sometimes over large distances (Koutrakis et al., 2007), resulted in the foundation of new stocks (Albrecht, 1983) and in the mixing of indigenous and non-indigenous populations. Over the last decades, the number of stocking events that disregard the genetic structure within and between populations (Souty-Grosset and Reynolds, 2009) and cross-basin translocations in response to rapidly 
declining stocks have led to a contamination of local stocks (Largiadèr et al., 2000). This has led to repeated calls for modern conservation programs that consider the genetic origin of the stocking material (e.g., Schulz et al., 2004; Bertocchi et al., 2008; Souty-Grosset and Reynolds, 2009; Strayer and Dudgeon, 2010).

Previous studies on noble crayfish taxonomy and biogeography are based solely on morphological characteristics (Bott, 1950, 1972; Karaman, 1962, 1963; Albrecht, 1983), while molecular studies have only focused on restricted geographical areas (Agerberg, 1990; Fevolden et al., 1994; Schulz, 2000; Edsman et al., 2002; Schulz et al., 2004; Alaranta et al., 2006). This study is the first molecular-based study of $A$. astacus covering a large portion of its distribution range, including river catchments of the North and Baltic Seas in central Europe and the Black Sea in southeastern Europe. The main aim of this study was to determine the degree of genetic structure in noble crayfish populations across three major catchments areas. In particular, we focused on asking to what extent this structure is the consequence of natural migration due to past climatic fluctuations during the Pleistocene or recent man-induced translocations. In the latter case, we would expect a lower genetic structure due to shared haplotypes between geographically disconnected populations. An anthropogenic influence could have caused a homogenization of haplotypes throughout Europe. The identification of inter-basin diversity could result in the conservation of catchment-specific gene pools (i.e., the identification of ESUs) to protect the present-day genetic diversity (Weiss et al., 2002). Finally, the results of this study are discussed in the context of conservation management plans for this vulnerable species.

\section{MATERIALS AND METHODS}

\section{$>$ SAMPLING}

A total of 416 crayfish specimens from 92 sampling locations (Figure 1, Tables I and II) were collected either by hand or with traps within catchments in the North, Baltic, and Black Seas. Immediately after the lower part of one pereopod (propodus and dactylus) was taken, specimens were released at the place where they were caught. Appendages usually regenerate after a few molts. The samples were stored in $96 \%$ ethanol until DNA extraction. DNA was extracted from the muscle tissue using a standardized protocol ("rapid isolation of Mammalian DNA", Sambrook and Russel, 2001).

\section{> LABORATORY PROCEDURES}

A 350 base pair (bp) fragment of the mitochondrial cytochrome oxidase subunit I (COI) was sequenced using the primer pair ASTCOI (forward primer: 5'-GCGGGGATAGTAGGAACCTC3'; reverse primer: 5'-ATTTACCGCCCCTAAAATCG-3'; Schrimpf and Schulz, in prep.). Polymerase chain reactions (PCR) were performed in a total volume of $25 \mu \mathrm{L}$ containing $0.625 \mathrm{u}$ GoTaq DNA Polymerase (Promega, Mannheim, Germany), $1 \times$ Colorless GoTaq ${ }^{\circledR}$ Flexi Buffer (Promega, Mannheim, Germany), 2 mM MgCl 2 (Promega, Mannheim, Germany), $0.24 \mathrm{mM}$ of each dNTP (Fermentas, St. Leon-Rot, Germany), $0.2 \mu \mathrm{M}$ of each primer and $20 \mathrm{ng}$ of template DNA. The PCR reaction was performed using a Primus 96 Cycler (Peqlab Biotechnologie $\mathrm{GmbH}$, Erlangen, Germany) under the following conditions: an initial 2 min step at $95{ }^{\circ} \mathrm{C}$, followed by 35 cycles with $45 \mathrm{~s}$ at $95^{\circ} \mathrm{C}, 45 \mathrm{~s}$ at $50^{\circ} \mathrm{C}, 1 \mathrm{~min}$ at $72{ }^{\circ} \mathrm{C}$, and a final extension of $5 \mathrm{~min}$ at $72{ }^{\circ} \mathrm{C}$. PCR products were stored at $10^{\circ} \mathrm{C}$. PCR products were partly sequenced on a 3730 DNA Analyzer eight capillary sequencer (Applied Biosystems, MA, USA) by the company SeqIT (Kaiserslautern, Germany). The remaining PCR product was purified with the AMPure XP system (Beckman Coulter, Krefeld, Germany). The fragments were amplified in a sequencing-reaction using the primer ASTCOIF and the CEQ DTCS-Quick Start kit (Beckman Coulter, Krefeld, Germany). After ethanol precipitation, sequencing was performed on a Beckman Coulter CEQ 8000 eight capillary sequencer. Each template was sequenced 

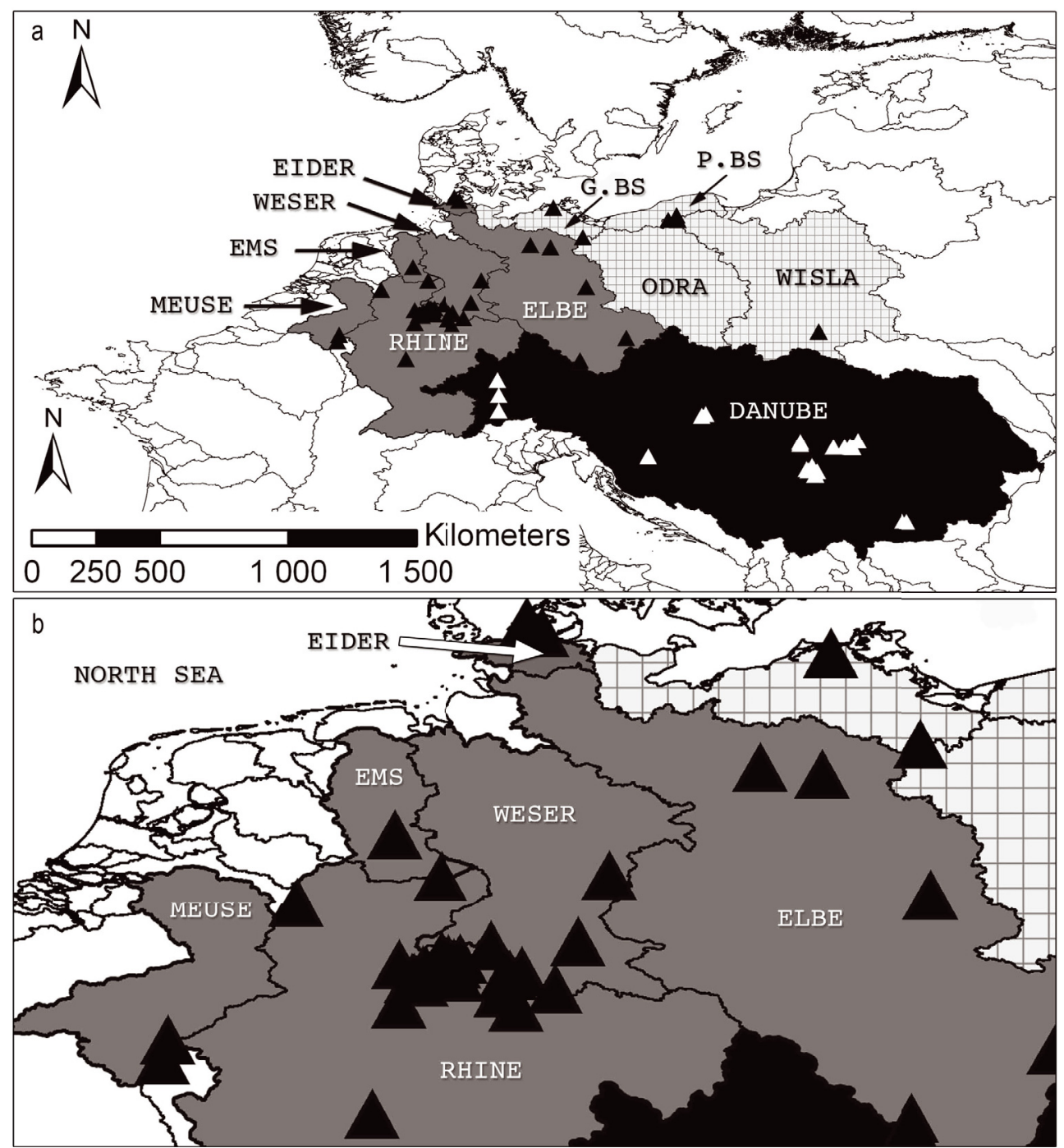

Figure 1

(a) Study area with sampling sites. Major catchment areas of European rivers are indicated by a checked pattern for the Baltic Sea catchment area; by dark grey for the North Sea catchment area; and by black for the Black Sea catchment area. G. BS = German Baltic Sea estuary, P. BS = Polish Baltic Sea estuary. (b) Larger scale map of the North Sea major catchment.

Figure 1

Aire d'étude des sites échantillonés. Les principaux bassins hydrographiques sont indiqués en gris clair pour le bassin de la Mer Baltique, en gris foncé pour le bassin de la Mer du Nord et en noir pour le bassin de la Mer Noire. G. BS = estuaire allemand en Mer Baltique; P. BS = estuaire polonais en Mer Baltique. (b) Carte à plus grande échelle du bassin versant de la Mer du Nord.

in the forward direction, and $50 \%$ of samples were randomly chosen for sequencing in the reverse direction.

The sequences were edited and aligned with the Sequencher 3.0 software (gene codes corporation, Ann Arbor, Michigan). The sequences were checked manually for base pair ambiguities, nuclear copies of mitochondrial derived genes, stop codons, and high levels of divergence, as recommended by Buhay (2009). All haplotypes were submitted to GenBank. 


\section{Table I}

Locality information of analyzed Astacus astacus populations. The originating countries and river catchments, the number of populations (Pop) and the number of sequenced individuals $(N)$, the number of haplotypes $\left(H_{N}\right)$, the haplotype diversity $\left(H_{\mathrm{D}}\right)$ and the number of polymorphic sites $(S)$ are given. Country Codes: $A U T=$ Austria; $B E L=$ Belgium: $B U L=$ Bulgaria; $C Z E=$ Czech Republic; GER = Germany; $C R O=$ Croatia; HUN = Hungary; $P O L=$ Poland; ROU = Romania .

\section{Tableau I}

Résumé des populations d'Astacus astacus analysées. Les pays d'origine, les bassins hydrographiques, le nombre de populations (Pop), le nombre d'individus séquencés $(N)$, le nombre d'haplotypes $\left(H_{N}\right)$, la diversité haplotypique $\left(H_{\mathrm{D}}\right)$ et le nombre de sites polymorphes $(\mathrm{S})$ sont indiqués. Codes pays $\mathrm{AUT}=\mathrm{Au}-$ triche, $\mathrm{BUL}=$ Bulgarie, $\mathrm{CZE}=$ République Tchèque, GER = Allemagne, $\mathrm{CRO}=$ Croatie, $\mathrm{HUN}=\mathrm{Hongrie}$, $\mathrm{POL}=$ Pologne, $\mathrm{ROU}=$ Roumanie

\begin{tabular}{|c|c|c|c|c|c|c|c|}
\hline $\begin{array}{c}\text { Major } \\
\text { catchment } \\
\text { area }\end{array}$ & Countries & River catchments & Pop & $N$ & $H_{N}$ & $H_{D}$ & $S$ \\
\hline Black Sea & $\begin{array}{c}\text { AUT, BUL, CRO, } \\
\text { GER, HUN, ROU }\end{array}$ & Danube & 30 & 106 & 16 & $0.794 \pm 0.024$ & 17 \\
\hline North Sea & CZE, GER, BEL & $\begin{array}{c}\text { Eider, Elbe, } \\
\text { Rhine, Meuse, } \\
\text { Weser }\end{array}$ & 50 & 241 & 11 & $0.299 \pm 0.038$ & 14 \\
\hline Baltic Sea & GER, POL & $\begin{array}{c}\text { Uecker, Odra, } \\
\text { Wisla, Wiprza }\end{array}$ & 16 & 69 & 3 & $0.163 \pm 0.058$ & 2 \\
\hline
\end{tabular}

\section{>STATISTICAL ANALYSES}

A median joining (MJ) network (Bandelt et al., 1999) was constructed using the software network 4.510 (www.Fluxus-Engineering.com, 2009) to identify haplotypes and to determine the phylogenetic relationships between haplotypes, which allows us to identify geographically localized haplogroups. The network was also calculated with TCS 1.21 (Clement et al., 2000) software to confirm the shape of the network.

Haplotype diversity $\left(H_{\mathrm{D}}\right)$ and the number of polymorphic sites $(S)$ per river catchment were calculated using the software ARLEQUIN 3.5.1 (Excoffier et al., 2005). The calculation of haplotype diversity is based on the frequency of a haplotype and the sample size (Nei, 1987). To detect genetic differentiation among major catchment areas, an analysis of the molecular variance (AMOVA, Excoffier et al., 1992), based on three hierarchical groups corresponding to the major catchment areas, and the pairwise $\Phi_{\mathrm{ST}}$-values (Weir and Cockerham, 1984) were calculated using the program ARLEQUIN 3.5.1. We further examined population genetic differentiation using exact tests of population differentiation (ETPD, Raymond and Rousset, 1995), as implemented in ARLEQUIN 3.5.1. In addition, $H_{\mathrm{D}}, S$, and pairwise $\Phi_{\mathrm{ST}}$-values (Weir and Cockerham, 1984) were calculated for sampling sites in the North Sea catchment area. Here, the Ems population has been excluded from the calculation of the pairwise $\Phi_{\mathrm{ST}}$-values due to the low sample size $(N=5)$.

\section{RESULTS}

$\mathrm{COI}$ sequences were analyzed for 416 specimens from 96 sample sites across three major catchment areas in Europe (Tables I and II, Figure 1). There were 20 variable sites within the 350 -bp fragment $(0.06 \%)$. Only four of these substitutions resulted in an amino acid change in four haplotypes (H04, H10, H21). In total, 23 unique haplotypes were detected (GenBank accession numbers: JN254659-JN254681), 17 of which were endemic to a specific river catchment. The overall haplotype diversity was $41.9 \%$. The median joining (MJ) network is shown in Figure 2. The shape was confirmed with the TCS software. The greatest observed distance between haplotypes was $9 \mathrm{bp}$. For the Black Sea catchment, 10 out of 16 haplotypes were endemic to this region. Of the 11 North Sea haplotypes, five were endemic to the catchment 
Table II

Sampling locations and haplotypes of analyzed Astacus astacus and the number of the most common haplotype (H01) and the number of all other haplotypes. WIE = Wieprza, WIS = Wisla, DA = Danube, $R H=$ Rhine, G. BS = German Baltic Sea estuary, P. BS = Polish Baltic Sea estuary, BAS = Baltic Sea, $B S=$ Black Sea, NS = North Sea. n.a. = not available. For country codes, see Table I.

Tableau II

Localisation de l'échantillonnage et haplotypes de Astacus astacus analysé et le nombre de l'haplotype le plus commun $(\mathrm{H01})$ et le nombre de tous les autres haplotypes. WIE = Wieprza, WIS $=$ Wisla, DA = Danube, $\mathrm{RH}=$ Rhin, G. BS = estuaire allemand de la Mer Baltique, P. BS = estuaire polonais de la Mer Baltique, BAS = Mer Baltique, BS = Mer Noire, NS = Mer du Nord. n.a. = non disponible. Pour les codes pays, voir Tableau I.

\begin{tabular}{|c|c|c|c|c|c|c|c|c|}
\hline Region & $\begin{array}{l}\text { Latitude } \\
\qquad\left(\mathrm{N}^{\circ}\right)\end{array}$ & $\begin{array}{c}\text { Longitude } \\
\left(\mathrm{E}^{\circ}\right)\end{array}$ & Country & \begin{tabular}{|c|} 
River \\
district
\end{tabular} & Estuary & $N$ & $\mathrm{H} 01$ & Haplotype \\
\hline Barthe & 54.333 & 12.833 & GER & G. BS & BAS & 5 & & $\mathrm{H} 02(5)$ \\
\hline Tonkuhle & 54.333 & 12.833 & GER & G. BS & BAS & 3 & 3 & \\
\hline Lake close to Miastko & 54.017 & 16.983 & $\mathrm{PO}$ & P. BS & BAS & 10 & 10 & \\
\hline Lake close to Miastko & 54.017 & 16.983 & $\mathrm{PO}$ & P. BS & BAS & 7 & 7 & \\
\hline Lake close to Miastko & 54.017 & 16.983 & $\mathrm{PO}$ & P. BS & BAS & 8 & 8 & \\
\hline Rakowe Duze & 53.915 & 16.796 & $\mathrm{PO}$ & Odra & BAS & 5 & 5 & \\
\hline Koppelsee & 53.307 & 13.855 & GER & Uecker & BAS & 3 & 3 & \\
\hline Seki & 54.065 & 17.095 & $\mathrm{PO}$ & WIE & BAS & 5 & 5 & \\
\hline Bez Nazwu & 54.067 & 17.083 & $\mathrm{PO}$ & WIE & BAS & 4 & 4 & \\
\hline Rosko & 53.903 & 17.143 & $\mathrm{PO}$ & WIS & BAS & 5 & 4 & $\mathrm{H} 03(1)$ \\
\hline Czarne & 50.038 & 21.993 & $\mathrm{PO}$ & WIS & BAS & 6 & 6 & \\
\hline Tomczyna & 50.036 & 22.004 & $\mathrm{PO}$ & WIS & BAS & 8 & 8 & \\
\hline Freundsheimer Weiher & 47.304 & 10.954 & $A$ & $\mathrm{DA}$ & BS & 3 & 2 & $\mathrm{H} 18(1)$ \\
\hline Razdvec & 43.463 & 24.908 & $\mathrm{BU}$ & DA & BS & 6 & 4 & $\mathrm{H} 13(1), \mathrm{H} 15(1)$ \\
\hline Beli Osam & 42.858 & 24.655 & $\mathrm{BU}$ & $\overline{D A}$ & BS & 3 & & $\mathrm{H} 14(3)$ \\
\hline Gorna Trape & 43.444 & 25.073 & $\mathrm{BU}$ & $\mathrm{DA}$ & BS & 6 & 1 & $\mathrm{H} 14(4), \mathrm{H} 16(1)$ \\
\hline Farm Augsburg & 48.359 & 10.906 & GER & $\overline{D A}$ & BS & 14 & 9 & $\begin{array}{c}\mathrm{H} 05(2), \mathrm{H} 06(1) \\
\mathrm{H} 2 \mathrm{O}(2)\end{array}$ \\
\hline Wielenbach & 47.841 & 10.952 & GER & $\mathrm{DA}$ & $\mathrm{BS}$ & 2 & 2 & \\
\hline Vukovina & 45.693 & 16.109 & $\mathrm{HR}$ & $\overline{D A}$ & BS & 5 & & $\mathrm{H} 17(5)$ \\
\hline Kádárta & 47.12 & 17.962 & $\mathrm{HU}$ & $\mathrm{DA}$ & BS & 2 & 2 & \\
\hline Pét & 47.184 & 18.096 & $\mathrm{HU}$ & $\mathrm{DA}$ & $\mathrm{BS}$ & 1 & & $\mathrm{H} 07(1)$ \\
\hline Clocotici & 45.241 & 21.838 & $\mathrm{RO}$ & $\mathrm{DA}$ & BS & 8 & & $\mathrm{H} 20(7), \mathrm{H} 12(1)$ \\
\hline Bradisoru de Jos & 45.104 & 21.768 & $\mathrm{RO}$ & $\overline{D A}$ & BS & 1 & & $\mathrm{H} 11(1)$ \\
\hline Carasova & 45.174 & 21.952 & $\mathrm{RO}$ & $\mathrm{DA}$ & BS & 1 & & $\mathrm{H} 11(1)$ \\
\hline Carasova & 45.176 & 21.943 & $\mathrm{RO}$ & DA & BS & 1 & & $\mathrm{H} 11(1)$ \\
\hline Garliste & 45.152 & 21.873 & $\mathrm{RO}$ & DA & BS & 5 & & $\mathrm{H} 11(5)$ \\
\hline Anina & 45.059 & 21.887 & $\mathrm{RO}$ & DA & BS & 2 & 2 & \\
\hline Ocna de Fier & 45.354 & 21.759 & $\mathrm{RO}$ & $\mathrm{DA}$ & BS & 4 & 1 & $\mathrm{H} 11(3)$ \\
\hline Forotic & 45.251 & 21.574 & $\mathrm{RO}$ & $\overline{D A}$ & BS & 6 & & $\mathrm{H} 11(6)$ \\
\hline Carnecea & 45.206 & 21.636 & $\mathrm{RO}$ & $\mathrm{DA}$ & BS & 1 & & $\mathrm{H} 11(1)$ \\
\hline Hartagani & 46.06 & 22.925 & $\mathrm{RO}$ & $\mathrm{DA}$ & $\mathrm{BS}$ & 1 & & $\mathrm{H} 21(1)$ \\
\hline Balsa & 46.019 & 23.12 & $\mathrm{RO}$ & DA & BS & 1 & 1 & \\
\hline Baita & 46.033 & 22.884 & $\mathrm{RO}$ & DA & BS & 2 & 2 & \\
\hline Bacaia & 46.01 & 23.171 & $\mathrm{RO}$ & DA & BS & 2 & 2 & \\
\hline Balsa & 46.04 & 23.07 & $\mathrm{RO}$ & DA & BS & 2 & 1 & $\mathrm{H} 20(1)$ \\
\hline Tamasesti & 46.022 & 22.507 & $\mathrm{RO}$ & $\overline{D A}$ & BS & 3 & 3 & \\
\hline Almasu Mic de Munte & 46.052 & 23.072 & $\mathrm{RO}$ & $\mathrm{DA}$ & BS & 3 & 2 & $\mathrm{H} 19(1)$ \\
\hline Cladova & 46.151 & 21.357 & $\mathrm{RO}$ & $\mathrm{DA}$ & $\mathrm{BS}$ & 4 & 2 & $\mathrm{H04(2)}$ \\
\hline Araneag & 46.21 & 21.357 & $\mathrm{RO}$ & $\mathrm{DA}$ & BS & 2 & 1 & $\begin{array}{l}\mathrm{H} 20(1) \\
\mathrm{H} 11(1)\end{array}$ \\
\hline
\end{tabular}


Table II

Continued.

Tableau II

Suite.

\begin{tabular}{|c|c|c|c|c|c|c|c|c|}
\hline Region & $\begin{array}{l}\text { Latitude } \\
\qquad\left(N^{\circ}\right)\end{array}$ & $\begin{array}{c}\text { Longitude } \\
\left(\mathrm{E}^{\circ}\right)\end{array}$ & Country & \begin{tabular}{|c|} 
River \\
district
\end{tabular} & Estuary & $N$ & $\mathrm{H} 01$ & Haplotype \\
\hline Nadas & 46.206 & 21.359 & $\mathrm{RO}$ & DA & BS & 14 & & $\mathrm{H} 12(1), \mathrm{H} 2 \mathrm{O}(12)$ \\
\hline Conop & 46.122 & 21.359 & $\mathrm{RO}$ & $\mathrm{DA}$ & BS & 1 & & $\mathrm{H} 20(1)$ \\
\hline Farm Oeversee, origin:Ls & 54.688 & 9.422 & GER & Eider & NS & 5 & & $\mathrm{H} 09(4), \mathrm{H} 10(1)$ \\
\hline Langsee (Ls) & 54.575 & 9.593 & GER & Eider & NS & 9 & & $\mathrm{H} 09(8), \mathrm{H} 10(1)$ \\
\hline Svetlohor & 48.977 & 13.736 & $\mathrm{CZ}$ & Elbe & NS & 7 & 7 & \\
\hline U sudu & 49.822 & 15.358 & $\mathrm{CZ}$ & Elbe & NS & 6 & 4 & $\overline{\mathrm{H} 18(2)}$ \\
\hline Kramata & 49.043 & 13.758 & $\mathrm{CZ}$ & Elbe & NS & 7 & 7 & \\
\hline Stepenitz & 53.037 & 12.042 & GER & Elbe & NS & 5 & 5 & \\
\hline Schwarze Elster & 51.582 & 13.97 & GER & Elbe & NS & 6 & 6 & \\
\hline Jäglitz & 52.96 & 12.741 & GER & Elbe & NS & 7 & 5 & $\mathrm{H} 17(2)$ \\
\hline Gut Rietberg & 51.802 & 8.435 & GER & Ems & NS & 5 & 4 & $\mathrm{H} 2 \mathrm{O}(1)$ \\
\hline Florenville & 49.700 & 5.307 & $\mathrm{BE}$ & Meuse & NS & 5 & 5 & \\
\hline Libramont & 49.927 & 5.371 & $\mathrm{BE}$ & Meuse & NS & 4 & 4 & \\
\hline n.a. & n.a. & n.a. & $\mathrm{BE}$ & Meuse & NS & 2 & 1 & $\mathrm{H} 12(1)$ \\
\hline Fischbach & 49.087 & 7.674 & GER & $\mathrm{RH}$ & NS & 6 & 3 & $\mathrm{H} 23(3)$ \\
\hline Klausbach & 52.272 & 7.910 & GER & $\mathrm{RH}$ & NS & 7 & 3 & $\mathrm{H} 2 \mathrm{O}(4)$ \\
\hline Mahlscheid & 50.778 & 7.972 & GER & $\mathrm{RH}$ & NS & 8 & 8 & \\
\hline Zoo Zajak, Petstore & 51.488 & 6.810 & GER & $\mathrm{RH}$ & NS & 4 & 4 & \\
\hline Aar & 50.675 & 8.487 & GER & $\mathrm{RH}$ & NS & 5 & 5 & \\
\hline Allna & 50.791 & 8.591 & GER & $\mathrm{RH}$ & NS & 4 & 4 & \\
\hline Ambach & 50.702 & 8.275 & GER & $\mathrm{RH}$ & NS & 5 & 5 & \\
\hline Mühlgraben Caldern & 50.842 & 8.658 & GER & $\mathrm{RH}$ & NS & 3 & 2 & $\mathrm{H05(1)}$ \\
\hline Dautphe & 50.838 & 8.525 & GER & $\mathrm{RH}$ & NS & 3 & 3 & \\
\hline TA Donsbach & 50.708 & 8.242 & GER & $\mathrm{RH}$ & NS & 5 & 5 & \\
\hline Eichelbach & 50.459 & 9.123 & GER & $\mathrm{RH}$ & NS & 5 & 5 & \\
\hline Fohnbach & 50.636 & 8.622 & GER & $\mathrm{RH}$ & NS & 5 & 5 & \\
\hline Gansbach & 50.804 & 8.409 & GER & $\mathrm{RH}$ & NS & 5 & 5 & \\
\hline TA Geierstein/ Roth & 50.641 & 8.226 & GER & $\mathrm{RH}$ & NS & 5 & 5 & \\
\hline Giebelsbach & 50.997 & 9.012 & GER & $\mathrm{RH}$ & NS & 1 & 1 & \\
\hline Kallenbach & 50.582 & 8.226 & GER & $\mathrm{RH}$ & NS & 10 & 10 & \\
\hline Lasterbach/ TA Mabüll & 50.591 & 8.142 & GER & $\mathrm{RH}$ & NS & 5 & 5 & \\
\hline Merzkrebse & 50.336 & 7.976 & GER & $\mathrm{RH}$ & NS & 9 & 9 & \\
\hline Meerbach & 50.706 & 8.482 & GER & $\mathrm{RH}$ & NS & 1 & 1 & \\
\hline Madenmühlen & 50.624 & 8.143 & GER & $\mathrm{RH}$ & NS & 5 & 5 & \\
\hline Mademühlen 2 & 50.624 & 8.143 & GER & $\mathrm{RH}$ & NS & 2 & 2 & \\
\hline Nanzenbach & 50.78 & 8.489 & GER & $\mathrm{RH}$ & NS & 1 & 1 & \\
\hline Perf & 50.873 & 8.459 & GER & $\mathrm{RH}$ & NS & 5 & 5 & \\
\hline TA Hartmann/ Rehbach & 50.624 & 8.120 & GER & $\mathrm{RH}$ & NS & 5 & 5 & \\
\hline Salzbach & 50.753 & 8.539 & GER & $\mathrm{RH}$ & NS & 5 & 5 & \\
\hline TA bei Spielberg & 50.304 & 9.298 & GER & $\mathrm{RH}$ & NS & 5 & 2 & $\mathrm{H} 20(3)$ \\
\hline Steinbruch Rot/ Schönbach & 50.654 & 9.219 & GER & $\mathrm{RH}$ & NS & 5 & 5 & \\
\hline Stippbach & 50.704 & 9.359 & GER & $\mathrm{RH}$ & NS & 5 & 5 & \\
\hline Pollichia Woog & 49.103 & 7.719 & GER & $\mathrm{RH}$ & NS & 2 & & $\mathrm{H} 20(2)$ \\
\hline Pollichia Woog2 & 49.103 & 7.719 & GER & $\mathrm{RH}$ & NS & 2 & 1 & $\mathrm{H} 2 \mathrm{O}(1)$ \\
\hline Schlettenbachtal, Fischbach & 49.088 & 7.711 & GER & $\mathrm{RH}$ & NS & 1 & & $\mathrm{H} 20(1)$ \\
\hline Wolfsägertal & 49.106 & 7.701 & GER & $\mathrm{RH}$ & NS & 7 & 2 & $\begin{array}{c}\mathrm{H} 08(1), \mathrm{H} 2 \mathrm{O}(2), \\
\mathrm{H} 22(2)\end{array}$ \\
\hline
\end{tabular}




\section{Table II}

Continued.

Tableau II

Suite.

\begin{tabular}{|l|c|c|c|c|c|c|c|c|}
\hline Region & $\begin{array}{c}\text { Latitude } \\
\left(\mathrm{N}^{\circ}\right)\end{array}$ & $\begin{array}{c}\text { Longitude } \\
\left(\mathrm{E}^{\circ}\right)\end{array}$ & Country & $\begin{array}{c}\text { River } \\
\text { district }\end{array}$ & Estuary & N & H01 & Haplotype \\
\hline Meisertalweiher & 49.335 & 7.769 & GER & $\mathrm{RH}$ & $\mathrm{NS}$ & 5 & 5 & \\
\hline Waldteich Irrschelde & 50.763 & 8.399 & GER & $\mathrm{RH}$ & $\mathrm{NS}$ & 5 & 5 & \\
\hline Waldteich bei Wallenfels & 50.774 & 8.438 & GER & $\mathrm{RH}$ & $\mathrm{NS}$ & 5 & 5 & \\
\hline Clausthal-Zellerfeld* & 51.809 & 10.352 & GER & Weser & $\mathrm{NS}$ & 1 & 1 & \\
\hline Breitweiher/Rhön & 50.507 & 9.730 & GER & Weser & $\mathrm{NS}$ & 2 & 2 & \\
\hline Ocherbach & 50.673 & 9.185 & GER & Weser & $\mathrm{NS}$ & 5 & 5 & \\
\hline Urff & 51.041 & 9.991 & GER & Weser & $\mathrm{NS}$ & 4 & 4 & \\
\hline
\end{tabular}

* This sequence was obtained from a preserved specimen of the Senckenberg Museum in Frankfurt (SMF 13095).

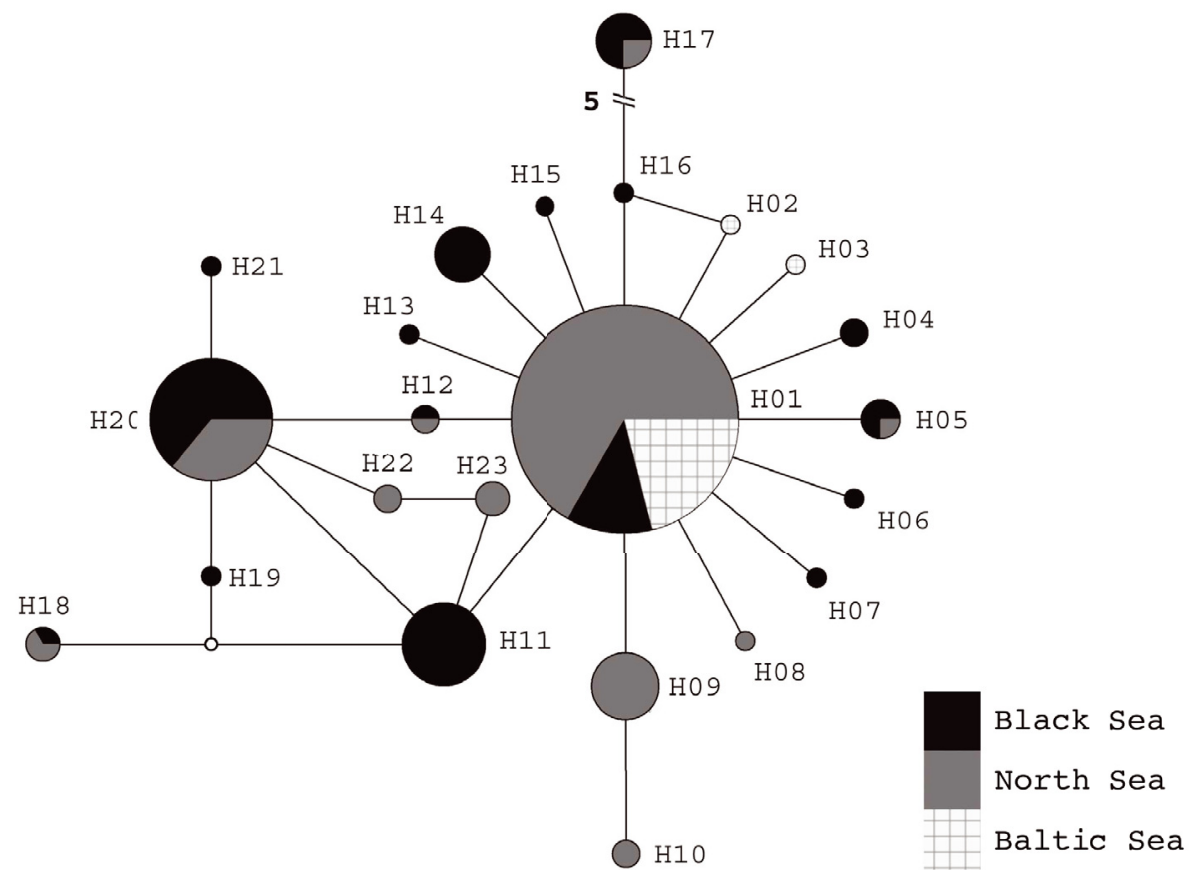

\section{Figure 2}

Median joining network of $\mathrm{CO}$ haplotypes (350 bp) from 416 individuals of Astacus astacus. The size of the circles is proportional to the frequency of the haplotypes. Median vectors are indicated as white dots. The number of base pair (bp) changes are given; no number $=1$ bp change.

\section{Figure 2}

Réseau d'haplotypes (350 pb) construit à partir de 416 individus d'Astacus astacus. La taille des cercles est proportionnelle à la fréquence des haplotypes. Les vecteurs médians sont indiqués par des points blancs. Le nombre de changements nucléotidiques est indiqué, aucun nombre $=$ un changement de $1 \mathrm{pb}$. 


\section{Table III}

Results of the analysis of molecular variance (AMOVA). Shown are the percentage of the total variance $\left(\%\right.$ Var), fixation indices $(F)$ and their significance $\left.{ }^{* * *}: p<0.001\right)$ based on 1000 random permutations.

\section{Tableau III}

Résultats de l'analyse de variance moléculaire (AMOVA). Les pourcentages de la variance totale (\% Var), les indices de fixation $(\mathrm{F})$ et leur significativité $\left(^{* * *}: p<0,001\right)$ basée sur 1000 permutations aléatoires sont donnés.

\begin{tabular}{|l|c|c|c|}
\hline Variance components & df. & $\%$ Var & F-statistics \\
\hline Among major catchment areas & 2 & 5.06 & $\Phi_{\mathrm{CT}}=0.051$ \\
\hline Among river catchments within major catchment areas & 9 & 23.92 & $\Phi_{\mathrm{SC}}=0.252^{\star \star \star}$ \\
\hline Within river catchments & 406 & 71.02 & $\Phi_{\mathrm{ST}}=0.290^{\star \star \star}$ \\
\hline
\end{tabular}

\section{Table IV}

Diversity values for the North Sea sampling area. The number of sequenced individuals (N), the number of haplotypes $\left(H_{\mathrm{N}}\right)$, the haplotype diversity $\left(H_{\mathrm{D}}\right)$ and the number of polymorphic sites $(S)$ for seven rivers flowing into the North Sea are given.

\section{Tableau IV}

Valeurs de diversité pour l'échantillonnage concernant la Mer du Nord. Le nombre d'individus séquencés $(N)$, le nombre d'haplotypes $\left(H_{N}\right)$, la diversité haplotypique $\left(H_{D}\right)$ et le nombre de sites polymorphes (S) sont indiqués pour sept affluents de la Mer du Nord.

\begin{tabular}{|l|c|c|c|c|}
\hline River catchment & $N$ & $H_{N}$ & $H_{\mathrm{D}}$ & $S$ \\
\hline Elbe & 38 & 3 & $0.199 \pm 0.084$ & 8 \\
\hline Ems & 5 & 2 & $0.400 \pm 0.237$ & 2 \\
\hline Meuse & 11 & 2 & $0.182 \pm 0.144$ & 1 \\
\hline Rhine & 161 & 6 & $0.227 \pm 0.043$ & 5 \\
\hline Weser & 12 & 1 & $0.000 \pm 0.000$ & 0 \\
\hline Eider & 14 & 2 & $0.264 \pm 0.136$ & 1 \\
\hline
\end{tabular}

area, and two out of three haplotypes were exclusively found in the rivers discharging into the Baltic Sea. Five haplotypes ( $\mathrm{H} 05, \mathrm{H} 12, \mathrm{H} 17, \mathrm{H} 18, \mathrm{H} 20)$ were shared between populations in the Black and North Seas. One haplotype $(\mathrm{H01})$, representing the center of the MJ network, was found frequently in all regions, resulting in a star-like topology of the network. A list of all haplotypes for each sampling site is given in Table II.

$H_{D}$-values (Table I) were lower for the rivers in central Europe (North Sea: $H_{D}=0.299 \pm 0.038$ and Baltic Sea: $\left.H_{D}=0.163 \pm 0.058\right)$ than for the Black Sea catchment $\left(H_{D}=0.794 \pm 0.024\right)$. Pairwise comparisons between river catchments revealed the lowest $\Phi_{\mathrm{ST}}$-value between the North and the Baltic Seas $\left(\Phi_{\mathrm{ST}}=0.035\right)$, and the highest value between the Black and Baltic Seas $\left(\Phi_{\mathrm{ST}}=0.204\right.$, Table V). The $\Phi_{\mathrm{ST}}$-value between the North Sea and the Black Sea was 0.161 . All values were highly significant $(p<0.001)$. The results of the AMOVA based on the three hierarchical groups (the major catchment areas) are given in Table III and reveal highly significant genetic differentiation between the major catchment areas $\left(\Phi_{\mathrm{ST}}=0.290\right.$, $p<0.001)$ and among river catchments within the major catchment areas $\left(F_{\mathrm{SC}}=0.252\right.$, $p<0.001)$.

$H_{\mathrm{D}}$-values for river catchments that open into the North Sea ranged between 0.000 (Weser) and 0.400 (Ems), although these minimum and maximum values could be due to low sample sizes (Weser: $N=12$; Ems: $N=5$; see Table IV). $H_{\mathrm{D}}$ for the Rhine $(N=161)$ was 0.227 . Pairwise $\Phi_{\mathrm{ST}}$-values for the North Sea major catchment area (Table $\mathrm{VI}$ ) revealed very low genetic differentiation between sampled rivers. However, the Eider exhibited a high differentiation compared to all of the other rivers of the North Sea catchment area $\left(\Phi_{\mathrm{ST}}\right.$-value between 0.575 and $0.876 ; p<0.001)$. 


\section{Table V}

Pairwise $\Phi_{\mathrm{ST}}-$ values between the three major catchment areas and their significance $\left(^{*}: p<0.05\right.$, $* * *: p<0.001)$.

\section{Tableau V}

Comparaison des valeur par paire $\Phi_{\mathrm{ST}}$ entre les trois principaux bassins versants et leur valeur significative $\left(^{*}: p<0,05,{ }^{* * *}: p<0,001\right)$.

\begin{tabular}{|c|c|c|c|}
\hline & Black Sea & North Sea & Baltic Sea \\
\hline Black Sea & 0 & & \\
\hline North Sea & $0.16131^{\star \star *}$ & 0 & \\
\hline Baltic Sea & $0.20387^{\star \star *}$ & $0.03481^{\star \star *}$ & 0 \\
\hline
\end{tabular}

\section{Table VI}

Pairwise $\Phi_{\mathrm{ST}}$-values between five rivers flowing into the North Sea and their significance $\left(^{*}: p<0.05\right.$, $* * *: p<0.001)$.

\section{Tableau VI}

Comparaison des valeur par paire $\Phi_{\mathrm{ST}}$ entre cinq rivières se jetant dans la Mer du Nord et leur valeur significative $\left(^{*}: p<0,05,{ }^{* *}: p<0,001\right)$.

\begin{tabular}{|l|c|c|c|c|c|}
\hline & Elbe & Meuse & Rhine & Weser & Eider \\
\hline Elbe & 0 & & & & \\
\hline Meuse & -0.011 & 0 & & & \\
\hline Rhine & $0.042^{\star}$ & -0.018 & 0 & & \\
\hline Weser & -0.016 & 0.008 & 0.007 & 0 & \\
\hline Eider & $0.575^{\star \star \star}$ & $0.816^{\star \star \star}$ & $0.699^{\star \star \star}$ & $0.876^{\star \star *}$ & 0 \\
\hline
\end{tabular}

\section{DISCUSSION}

This is the first large-scale molecular study of Astacus astacus. The main aim was to determine the degree and origin (natural migration versus artificial translocation) of the genetic structure in noble crayfish populations across three major catchment areas.

We detected very low haplotype diversities in noble crayfish populations in the North Sea and Baltic Sea catchments, with the exception of the Eider at the very northern end of Germany. The observed significant structure ( $\Phi_{\mathrm{ST}}$-values between 0.575 and 0.876$)$ between the Eider and all other North Sea river catchments may be explained by an isolation of the Eider population. The Eider exhibits an east to west current, which is in contrast to the primarily south-north running rivers of southern Germany. This might result in a disconnection of the Eider from the other southern river systems, where the catchment areas of streams partly overlap and gene flow seems more likely. As a peripheral source with restricted gene flow, the Eider population contributes to the overall genetic diversity within $A$. astacus. To discover the origin of the private haplotypes solely found within this river catchment (indigenous or non-indigenous), additional populations nearby should be analyzed.

Noble crayfish populations within the central European major catchment areas are significantly less differentiated than within the Black Sea catchment (Table I). A high haplotype diversity and a high number of private haplotypes were detected for the Black Sea populations, suggesting a glacial refuge of the noble crayfish in the Balkan area. During the Pleistocene, severe climatic fluctuations in central Europe resulted in repeated range expansions and regressions of many taxa. The distribution of freshwater species was especially influenced by the fluctuating glaciers and varying water levels, which resulted in landscape changes and modified river systems (Hänfling et al., 2009). The re-colonization of previously glaciated habitats (northern Germany) could have caused successive bottlenecks (Taberlet et al., 1998), which could explain the loss of genetic diversity and the observed dominance of haplotype $\mathrm{H} 01$ in the North Sea and Baltic Sea major catchments. A decrease in genetic diversity from southern to northern populations has been observed for many freshwater species, for example, 
the freshwater fish Vimba vimba (Hänfling et al., 2009) or the mayfly Ameletus inopinatus (Taubmann et al., 2011). The prevalence of haplotype H01 in the entire study area (Figure 1) may indicate a re-colonization event from the Balkan area to central Europe. The fact that the North Sea and Baltic Sea major catchments only share one haplotype and show some degree of differentiation (Tables II and V) may be interpreted as a result of two routes of recolonization from a common refuge. One migratory pathway into central Europe may have been the Danube drainage system after deglaciation, as presumed by Schulz and Grandjean (2005). The Danube represents a widely used corridor of postglacial expansion into central Europe for many freshwater species for which the possibility of a large-scale terrestrial dispersal has been excluded (e.g., Durand et al., 1999; Weiss et al., 2002; Hänfling et al., 2009). A second re-colonization route, which would explain the divergence of the North Sea and Baltic Sea major catchments, cannot be confirmed due to the lack of sample sites from eastern Europe. The occurrence of noble crayfish in the Baltic Sea major catchment may also be the result of human translocation, although no evidence exists to support this theory.

Our study indicates that the original distribution pattern of the noble crayfish has changed significantly due to anthropogenic influence (Skurdal and Taugbøl, 2002), resulting in a mixture of indigenous and non-indigenous populations (Largiadèr et al., 2000). Endemic haplotypes of the North Sea and Baltic Sea catchments could be the result of artificial stocking, or using stock from a river catchment not included in our study (e.g., from Russia). However, presumably no stocking of native crayfish has been conducted in the Balkan States and the Ponto-Caspian region (Albrecht, 1983). We can therefore assume a natural origin of the studied populations from the Balkans (Maguire, 2009). The high frequency of private haplotypes in all major catchment areas, as well as the relatively high genetic differentiation between the catchment areas ( $\Phi_{\mathrm{ST}}$-values between 0.035 and 0.204$)$, indicates a differentiation of populations throughout Europe despite the extensive human translocation of noble crayfish. Nevertheless, the artificial translocation of individuals can be observed in the disjunct distribution of some shared haplotypes between different drainage areas (Figure 1). Haplotype $\mathrm{H} 20$ could be an example of artificial distribution across central Europe. This haplotype was detected in the lower section of the Danube (Black Sea catchment), in the river catchments of the Rhine and Ems, and in individuals from a crayfish farm in Augsburg, Southern Germany (Table VII). This farm was founded by mixing four donor populations of the upper section of the Danube catchment area (Keller, pers. com.). Because restocking programs have been frequently conducted throughout Germany over the last few decades, in particular using stocking materials from the above-mentioned farm in Augsburg (Keller, 1999; Souty-Grosset and Reynolds, 2009), it is conceivable that specimens carrying haplotype $\mathrm{H} 20$ may originate from the Danube area and were artificially spread over central Europe. Human impact is also assumed to affect the (haplotype-) distribution of other European crayfish species, such as Austropotamobius pallipes (e.g., Grandjean et al., 2001; Diéguez-Uribeondo et al., 2008) or Austropotamobius italicus (Cataudella et al., 2010). Until today, the natural migratory routes of these species could not be fully inferred. However, the authors revealed geographical differences in the haplotype distribution and diversity, which may influence the conservation management strategies for these species.

With regard to $A$. astacus, the high frequency $(74 \%)$ of private haplotypes in the Black Sea catchment highlights the importance of the Balkans as a reservoir for intraspecific genetic diversity. In addition to the topographical complexity, which allows for the isolation of single populations on a small geographical scale, the Balkans were relatively unaffected by geological events during the glacial cycles. Thus, biodiversity is more conserved in the Balkans compared to central Europe for many species (Trontelj et al., 2005; Hänfling et al., 2009). Therefore, we strongly suggest a definition of several distinct ESUs along the Black Sea major catchment area. To define such ESUs, more populations of the Black Sea catchment area have to be analyzed because there might be much higher levels of genetic diversity and more detectable, endemic haplotyes to be found, for example, in the Upper Kolpa drainage similar to the recently detected distinct clades of Austropotamobius torrentium (Trontelj et al., 2005). So far, we suggest avoiding the intermixing of noble crayfish populations between the 
tributaries of the Danube. This is especially important for countries that plan to found crayfish farms for potential future restocking programs, e.g., in Bulgaria (Zaikov and Hubenova, 2007). We strongly emphasize that haplotype identification of the donor population should be conducted before a breeding stock is used for restocking. Additionally, the waters to be restocked should be carefully selected to avoid the contamination of local stocks.

If restocking programs continue to translocate individuals with no regard for their population's genetic structure, the natural genetic make-up will further dissolve (Souty-Grosset et al., 2003), which is accompanied by a reduction in intraspecific diversity. Conservation strategies, therefore, need to manage populations of $A$. astacus as distinct ESUs and give the highest priority to the populations with high genetic diversity and unique haplotypes. Mitochondrial DNA is a widely used marker to reconstruct the phylogeographic history of species. Here, we show that the analysis of partial $\mathrm{COI}$-sequences helps to understand the genetic structure of noble crayfish. However, the variation in noble crayfish COl-sequences is relatively low and the resolution is limited. Additional DNA-sequences should be included in the analysis to increase the resolution. Finally, a microsatellite analysis should be conducted to estimate genetic diversity within populations to give special attention to diverse populations and to further identify artificially stocked and naturally dispersed populations. However, as long as we cannot reject the hypothesis that private central European haplotypes developed due to local adaption, those populations that harbor endemic haplotypes are suggested to be candidates for special management plans to prevent the loss of unique haplotypes and to protect overall genetic diversity.

In conclusion, the results of this study suggest a glacial refuge in the Balkan area and a postglacial re-colonization of central Europe. Despite human translocations, which were revealed by the disjunct distribution of some haplotypes, a differentiation of noble crayfish populations in all major catchment areas supports the establishment of distinct ESUs to protect its present-day genetic diversity in Europe.

\section{ACKNOWLEDGEMENTS}

We thank the following colleagues for providing samples: Erik Bohl, Carsten Burk, Christoph Dümpelmann, Gerhard Feldhaus, Harald Gross, Didier Herman, Tania Hubenova, Max Keller, Helmut Jeske, Goran Klobučar, Pavel Kozák, Julia Langer, Andreja Lucic, Frank Meis, Ines Podszuck, Ronald Polivka, Miklós Puky, Przemyslaw Śmietana, Steffen Zahn, and the Senckenberg Natural History Museum in Frankfurt. We are very grateful to Therese Bürgi, Jennifer Dahlem, and Kathrin Metzner for help in the laboratory. The study was financially supported by the Bundesministeriums für Ernährung, Landwirtschaft und Verbraucherschutz (BMELV) through the Bundesanstalt für Landwirtschaft und Ernährung (BLE).

\section{REFERENCES}

Abrahamsson S.A.A., 1971. Density, growth and reproduction in populations of Astacus astacus and Pacifastacus leniusculus in an isolated pond. Oikos, 22, 373-380.

Agerberg A., 1990. Genetic variation in three species of freshwater crayfish; Astacus astacus L., Astacus leptodactylus Aesch., and Pacifastacus leniusculus (Dana), revealed by isozyme electrophoresis. Hereditas, 113, 101-108.

Alaranta A., Henttonen P., Jussila J., Kokko H., Prestegaard T., Edsman L. and Halmekyto M., 2006. Genetic differences among noble crayfish (Astacus astacus) stocks in Finland, Sweden and Estonia based on the ITS1 region. Bull. Fr. Pêche Piscic., 380-381, 965-975.

Albrecht H., 1983. Besiedlungsgeschichte und ursprüngliche holozäne Verbreitung der europäischen Flußkrebse (Decapoda: Astacidae). Spixiana, 6, 61-77.

Alderman D.J., 1996. Geographical spread of bacterial and fungal diseases of crustaceans. Rev. Sci. Tech. Off. Int. Epizoot., 15, 603-632. 
Alderman D.J., Holdich D. and Reeve I., 1990. Signal crayfish as vectors in crayfish plague in Britain. Aquaculture, 86, 3-6.

Bandelt H.-J., Forster P. and Röhl A., 1999. Median-joining networks for inferring intraspecific phylogenies. Mol. Biol. Evol., 16, 37-48.

Bertocchi S., Brusconi S., Gherardi F., Grandjean F. and Souty-Grosset C., 2008. Genetic variability of the threatened crayfish Austropotamobius italicus in Tuscany (Italy): implications for its management. Fund. App. Lim., 173, 153-164.

Bott R., 1950. Die Flusskrebse Europas (Decapoda, Astacidae). Abh. Senckenberg. Naturf. Ges., 483, $1-36$.

Bott R., 1972. Besiedlungsgeschichte und Systematik der Astaciden West-Europas unter besonderer Berücksichtigung der Schweiz. Rev. Suisse Zool., 79, 387-408.

Buhay J.E., 2009. "COI-like" sequences are becoming problematic in molecular systematic and DNA barcoding studies. J. Crustacean Biol., 29, 96-110.

Cataudella R., Paolucci M., Delaunay C., Ropiquet A., Hassanin A., Balsamo M. and Grandjeand F., 2010. Genetic variability of Austropotamobius italicus in the Marches region: implications for conservation. Aquatic Conserv. Mar. Freshw. Ecosyst. 20, 261-268.

Clement M., Posada D. and Crandall K.A., 2000. TCS: a computer program to estimate gene genealogies. Mol. Ecol., 9, 1657-1660.

Convention on Biological Diversity. Concluded at Rio de Janeiro on 5 June 1992, http://www.cbd.int/, accessed December 2010.

Crozier R.H., 1997. Preserving the information content of species: genetic diversity, phylogeny and conservation worth. Annu. Rev. Ecol. Syst., 28, 243-268.

Diéguez-Uribeondo J., Royo F., Souty-Grosset C., Ropiquet A. and Grandjean F., 2008. Low genetic variability of the white-clawed crayfish in the lberian Peninsula: its origin and management implications. Aquat. Conserv. Mar. Freshwat. Ecosyst., 18, 19-31.

Durand J.D., Persat H. and Bouvet Y., 1999. Phylogeography and postglacial dispersion of the chub (Leuciscus cephalus) in Europe. Mol. Ecol., 8, 989-997.

Edsman L., Farris J.S., Kallersjo M. and Prestegaard T., 2002. Genetic differentiation between noble crayfish, Astacus astacus (L.) populations detected by microsatellite length variation in the rDNA ITS1 region. Bull. Fr. Pêche Piscic., 367, 691-706.

Edsman L., Füreder L., Gherardi F. and Souty-Grosset C., 2010. Astacus astacus. In: IUCN 2010, IUCN Red List of Threatened Species, Version 2010.4, http://www.iucnredlist.org, accessed December 2010.

Excoffier L., Smouse P.E. and Quattro J.M., 1992. Analysis of molecular variance inferred from metric distances among DNA haplotypes: application to human mitochondrial-DNA restriction data. Genetics, 131, 479-491.

Excoffier L., Laval G. and Schneider S., 2005. Arlequin version 3.11: An integrated software package for population genetics data analysis. Evol. Bioinform. Online, 1, 47-50.

Fevolden S.E., Taugbøl T. and Skurdal J., 1994. Allozymic variation among populations of noble crayfish, Astacus astacus L., in southern Norway: implications for management. Aquacult. Fish. Manage., 25, 927-935.

Grandjean F., Gouin N., Souty-Grosset C. and Diéguez-Uribeondo J., 2001. Drastic bottlenecks in the endangered crayfish species, Austropotamobius pallipes in Spain with inference to its colonization history. Heredity, 88, $1-8$.

Hänfling B., Dümpelmann C., Bogutskaya N.G., Brandl R. and Brandle M., 2009. Shallow phylogeographic structuring of Vimba vimba across Europe suggests two distinct refugia during the last glaciation. J. Fish Biology, 75, 2269-2286.

Karaman M.S., 1962. Ein Beitrag zur Systematik der Astacidae (Decapoda). Crustaceana, 3, 173-191.

Karaman M.S., 1963. Studie der Astacidae (Crustacea, Decapoda) II. Teil. Hydrobiologia, 22, 111-132.

Keller M., 1999. Ten Years of Trapping Astacus astacus for Restocking in Lake Bronnen, a Gravel Pit in Bavaria. Freshwater Crayfish, 12, 518-528.

Koutrakis E., Perdikaris C., Machino Y., Savvidis G. and Margaris N., 2007. Distribution, recent mortalities and conservation measures of crayfish in hellenic freshwaters. Bull. Fr. Pêche Piscic., 385, 25-44. 
Largiadèr C.R., Herger F., Lörtscher M. and Scholl A., 2000. Assessment of natural and artificial propagation of the white-clawed crayfish (Austropotamobius pallipes species complex) in the Alpine region with nuclear and mitochondrial markers. Mol. Ecol., 9, 25-37.

Maguire I., 2009. Die Flusskrebse Osteuropas. In: Füreder L. (ed.), Flusskrebse, Biologie - Ökologie Gefährdung, Folio Verlag, Wien - Bozen, 92-97.

Moritz C., 1994. Defining 'evolutionary significant units' for conservation. Trends Ecol. Evol., 9, 373-375.

Nei M., 1987. Molecular Evolutionary Genetics, Columbia University Press, New York.

Östergren J., 2006. Migration and genetic structure of Salmo salar and Salmo trutta in northern Swedish rivers, Doctoral dissertation, Dept. of Aquaculture, SLU, Acta Universitatis agriculturae Sueciae.

Pullin A.S., Knight T.M., Stone D.A. and Charman K., 2004. Do conservation managers use scientific evidence to support their decision-making? Biol. Conserv., 119, 245-252.

Raymond M. and Rousset F., 1995. An exact test for population differentiation. Evolution 49, 1280-1283.

Ryder O.A., 1986. Species conservation and systematics: the dilemma of subspecies. Trends Ecol. Evol., 1, 9-10.

Sambrook J. and Russell D.W., 2001. Molecular cloning: a laboratory manual, Cold Spring Harbor Laboratory Press, New York.

Schikora F., 1906. Die Krebspest. Fischerei-Zeitung, 9, 529-532; 549-553; 561-566; 581-583.

Schulz H.K. and Grandjean F., 2005. Roundtable session 3: Phylogeny of European crayfish - improving the taxonomy of European crayfish for a better conservation. Bull. Fr. Pêche Piscic., 376-377, 829-836.

Schulz H.K., Smietana P. and Schulz R., 2004. Assessment of DNA variations of the noble crayfish (Astacus astacus L.) in Germany and Poland using inter-simple sequence repeats (ISSRs). Bull. Fr. Pêche Piscic., 372-373, 387-399.

Schulz R., 2000. Status of the noble crayfish Astacus astacus (L.) in Germany: monitoring protocol and the use of RAPD markers to assess the genetic structure of populations. Bull. Fr. Pêche Piscic., 356, 123-138.

Skurdal J. and Taugbøl T., 2002. Astacus. In: Holdich D.M. (ed.), Biology of Freshwater Crayfish, Blackwell Science Ltd, Oxford, 467-510.

Smietana P., Schulz H.K., Keszka S. and Schulz R., 2006. A proposal for accepting Pontastacus as a genus of European crayfish within the family Astacidae based on a revision of the west and east European taxonomic literature. Bull. Fr. Pêche Piscic., 380-381, 1041-1052.

Souty-Grosset C. and Reynolds J.D., 2009. Current ideas on methodological approaches in European crayfish conservation and restocking procedures. Knowl. Managt. Aquatic Ecosyst., 394-395, 01.

Souty-Grosset C., Grandjean F. and Gouin N., 2003. Involvement of genetics in knowledge, stock management and conservation of Austropotamobius pallipes in Europe. Bull. Fr. Pêche. Piscic., 370371, 165-179.

Strayer D.L. and Dudgeon D., 2010. Freshwater biodiversity conservation: recent progress and future challenges. J. N. Am. Benthol. Soc., 29, 344-358.

Taberlet P., Fumagalli L., Wust-Saucy A.-G. and Cosson J.-F., 1998. Comparative phylogeography and postglacial colonization routes in Europe. Mol. Ecol., 7, 453-464.

Taubmann J., Theissinger K., Feldheim K., Laube I., Graf W., Johannesen J., Haase P. and Pauls S.U., 2011. Modelling range shifts and assessing genetic diversity distribution of the montane aquatic mayfly Ameletus inopinatus in Europe under climate change scenarios. Conserv. Genet., 12, 503515.

Trontelj P., Machino Y. and Sket B., 2005. Phylogenetic and phylogeographic relationships in the crayfish genus Austropotamobius inferred from mitochondrial COI gene sequences. Mol. Phylogenet. Evol. 34, 212-226.

Weir B.S and Cockerham C.C., 1984. Estimating F-statistics for the analysis of population structure. Evolution, 38, 1358-1370.

Weiss S., Persat H., Eppe R., Schlötterer C. and Uiblein F., 2002. Complex patterns of colonization and refugia revealed for European grayling Thymallus thymallus, based on complete sequencing of the mitochondrial DNA control region. Mol. Ecol., 11, 1393-1407.

Zaikov A. and Hubenova T., 2007. Status of freshwater crayfish in Bulgaria. Proceedings III International conference "Fishery", 1-3 February 2007, Belgrade, 242-247. 